\title{
Analyzing the learning curves of a novice and an experienced urologist for transrectal magnetic resonance imaging-ultrasound fusion prostate biopsy
}

\author{
Emanuel Darius Cata ${ }^{1,2}$, Charles Van Praet ${ }^{3}$, Iulia Andras ${ }^{1,2}$, Pierre Kadula ${ }^{2}$, Razvan Ognean ${ }^{1}$, \\ Maximilian Buzoianu ${ }^{1}$, Daniel Leucuta ${ }^{4}$, Cosmin Caraiani ${ }^{5}$, Attila Tamas-Szora ${ }^{6}$, Karel Decaestecker ${ }^{3}$, \\ Ioan Coman ${ }^{1,2}$, Nicolae Crisan ${ }^{1,2}$ \\ ${ }^{1}$ Urology Department, Iuliu Hatieganu University of Medicine and Pharmacy, Cluj-Napoca, Romania; ${ }^{2}$ Urology Department, Municipal Hospital, \\ Cluj-Napoca, Romania; ${ }^{3}$ Urology Department, Ghent University Hospital, Ghent, Belgium; ${ }^{4}$ Medical Informatics and Biostatistics Department, Iuliu \\ Hatieganu University of Medicine and Pharmacy, Cluj Napoca, Romania; ${ }^{5}$ Medical Imaging Department, Iuliu Hatieganu University of Medicine \\ and Pharmacy, Cluj Napoca, Romania; ${ }^{6}$ Radiology Department, Municipal Hospital, Cluj Napoca, Romania \\ Contributions: (I) Conception and design: ED Cata, C Van Praet, I Andras, N Crisan; (II) Administrative support: C Van Praet, I Andras, D Leucuta, \\ N Crisan; (III) Provision of study materials or patients: ED Cata, IAndras, C Caraiani, A Tamas-Szora, N Crisan; (IV) Collection and assembly of \\ data: ED Cata, I Andras, P Kadula, R Ognean, M Buzoianu; (V) Data analysis and interpretation: ED Cata, C Van Praet, I Andras, P Kadula, D \\ Leucuta, K Decaestecker, N Crisan; (VI) Manuscript writing: All authors; (VII) Final approval of manuscript: All authors. \\ Correspondence to: Iulia Andras, MD, PhD, FEBU. Urology Department, Iuliu Hatieganu University of Medicine and Pharmacy Cluj-Napoca, 8 V \\ Babes, 400012, Cluj-Napoca, Romania. Email: dr.iuliaandras@gmail.com.
}

Background: The aim of the current study was to evaluate and compare the learning curves of transrectal magnetic resonance imaging-ultrasound fusion biopsy for two urologists with different backgrounds (Operator 1: experienced, self-trained and Operator 2: novice, trained by a mentor/MRI reading courses).

Methods: A cohort of 400 patients who underwent fusion prostate biopsy in our department was analyzed. The learning curves were assessed in terms of overall and clinically significant prostate cancer (PCa) detection rates, percentage of positive biopsy cores/targeted and the percentage of PCa tissue on positive targeted cores.

Results: Increasing trends were observed for both urologists in terms of all biopsy outcomes during the study time. For the novice urologist, a significant increase was observed for overall PCa detection rate, but not for clinically significant disease $(25.44 \%, \mathrm{P}=0.04 / 15 \%, \mathrm{P}=0.145)$. Operator 1 showed an increasing diagnosis yield of clinically significant disease up to 104 cases. Similar cancer detection rates were observed when comparing the first and last biopsies performed by both operators. Multivariate analysis adjusted for age, PSA, prostate volume, lesion diameter and PIRADS score showed an increase of PCa detection with $51 \%$ for every 52 biopsies performed $(\mathrm{P}=0.022)$.

Conclusions: When starting with magnetic resonance imaging-ultrasound fusion prostate biopsy, mentoring and prostate magnetic resonance imaging reading training allow a novice urologist to demonstrate a good initial PCa detection rate. After about 52 cases, he reached a stable PCa and clinically significant PCa detection rate, that was similar to that of an experienced urologist.

Keywords: Learning curve; magnetic resonance imaging; MRI-US fusion prostate biopsy; prostate cancer (PCa)

Submitted Jan 05, 2021. Accepted for publication Mar 19, 2021.

doi: $10.21037 /$ tau-21-8

View this article at: http://dx.doi.org/10.21037/tau-21-8 


\section{Introduction}

Prostate multiparametric magnetic resonance imaging (mpMRI) followed by both targeted and systematic biopsy is currently the gold standard pathway for men at risk of prostate cancer (PCa) (1). Three modalities are employed to perform targeted biopsy: cognitive, MRI-ultrasound (US) fusion and in-bore MRI biopsy.

Overall, the MRI-US fusion biopsy devices seem to be the most frequently used, with the number of fusion platforms available on the market increasing considerably in the last years. A recent report showed that $72 \%$ of the urologists currently employ such a system (2). The MRI-US fusion biopsy platforms have multiple advantages: real-time overlay of the MRI and ultrasound images, with precision similar to the in-bore targeted biopsy (3), possibility of concurrent systematic sampling, shorter duration of the procedure $v s$. in-bore sampling (4), increased patient compliance and less pain when compared to either in-bore or systematic biopsy $(4,5)$ and better cost-effectiveness from a healthcare system point of view $(6,7)$.

On the other hand, performing MRI-US fusion biopsy presents several challenges for the urologist: it requires knowledge of MRI interpretation and special training in MRI reading (8), together with the ability to manage and overcome potential sources of error such as lesion delineation, estimation of dimension of target lesion (9), patient movement, prostate deformation (10).

Despite all these limitations, several authors reported statistically significant improvement of PCa detection rate by MRI-US fusion biopsy over time [Meng et al. (11): $26 \%$ improvement after 1,700 procedures, $\mathrm{P}<0.05$; Calio et al. (12): $11.6 \%$ improvement after 1,728 procedures, $\mathrm{P}<0.05]$. Multiple reasons have been proposed to explain the rise of diagnosis rate in time such as: the learning curve with the subsequent experience, the proper training of the urologists or the better understanding and control of new technology. Whatever the arguments, factors that influence targeted biopsy performance need to be understood better for further improvement of the technique and development of standardized training programs.

The aim of the current study was to evaluate the learning curve for MRI-transrectal US (TRUS) fusion biopsy and to compare the differences in technique acquisition and biopsy outcome between two operators with different backgrounds and experience. We present the current article in accordance with the STROBE reporting checklist (available at http:// dx.doi.org/10.21037/tau-21-8).

\section{Methods}

\section{Patients}

A cohort of 410 consecutive patients underwent MRITRUS fusion guided prostate biopsy at a single academic institution between October 2017 and March 2020. Data was collected in a prospective manner. The study was conducted in accordance with the Declaration of Helsinki (as revised in 2013) and was approved by the local Hospital Ethical Committee (approval no. 8/20.02.2017). All patients enrolled completed and signed the informed consent form.

\section{Imaging and biopsy protocol}

All patients included in the analysis underwent mpMRI prior to the biopsy and harbored at least one suspicious area of PCa (region of interest - ROI). The current study aimed to reflect real-world practice, thus we included patients with mpMRI performed in different centers (129 mpMRIs from our centre, $271 \mathrm{mpMRIs}$ from external centers). In our centre, mpMRI were interpreted by 3 radiologists with more than 5 years of experience. The majority of mpMRI were performed on $1.5 \mathrm{~T}$ scanners with or without endorectal coil at radiologists' discretion. The protocol and acquisition parameters for our institution were previously described (13). The minimal requirements for including patients with external MRI were multiparametric scan including T2 weighted imaging, diffusion weighted imaging, dynamic contrast enhancement and at least $1.5 \mathrm{~T}$ magnetic field strength, together with a written report from the radiologist locating the ROI. The radiologists were aware of the patient's clinical information. The MRI interpretation was heterogenous, respecting either Prostate Imaging-Reporting And Data System (PIRADS) v1 or the updated PIRADS v2.1 criteria, using a five-point scale for both. For men with multiple ROI, the highest PIRADS score was considered the overall score of the patient.

All patients underwent MRI-TRUS fusion guided prostate biopsy using the Arrietta 70 system from Hitachi with RVS software and endfire endorectal probe. The overlay of the images was based on a rigid registration system. The urologist performed the delineation of the ROI based on radiologist drawing and/or description. The landmark used for the image fusion was the urethral axis. In order to account for a potential prostate deformation during the procedure, the delineation of the anterior and posterior prostate surface using two lines was performed 
in the plane of maximum diameter of the ROI. All biopsies were performed with the patients in left lateral decubitus and local anesthesia by endorectal instillation of a lidocaine gel. A magnetic field transmitter was positioned in front of the patient and a sensor on the endorectal probe, allowing for real-time biopsy tracking during the procedure.

MRI-TRUS fusion guided biopsies began with 1-4 targeted cores/ROI, followed by 12-core systematic biopsy. All procedures were performed by one of two urologists. Operator 1 (I.A.) had extensive experience in TRUSguided prostate biopsy, basic knowledge of interpretation of prostate mpMRI and started performing MRI-TRUS guided biopsies by self-teaching based on equipment manual and under the instruction of an application expert from the manufacturer. Operator 1 performed the first 209 procedures. Operator 2 (E.D.C.) started with minimal experience in TRUS guided procedures. He completed the European School of Urology course of mpMRI interpretation for urologists. He assisted at least 100 cases of MRI-TRUS fusion guided prostate biopsy and performed under the supervision of Operator 1 a number of 10 cases, which were excluded from the current analysis, due to the fact that we aimed to include only biopsies performed without supervision. After this experience, Operator 2 performed MRI-TRUS fusion biopsies independently. This cohort thus includes 400 patients and illustrates the learning curves of both urologists.

\section{Outcomes definition}

The learning curve was assessed as overall and clinically significant $\mathrm{PCa}$ (csPCa) diagnostic yield, percentage of positive cores/targeted and the percentage of $\mathrm{PCa}$ tissue on positive targeted cores. Clinically significant $\mathrm{PCa}$ was defined as ISUP grade 2 or higher.

\section{Statistical analysis}

The statistical analysis was performed using $\mathrm{R}$ programme v.3.6.2 on a per-patient-basis. The variables were presented as median and interquartile range (if continuous) or as frequencies (if categorical). Chi-square test was used to compare differences between categorical variables and Kruskall-Wallis test for the analysis of variance of continuous variables. $\mathrm{P}<0.05$ was considered statistically significant.

\section{Results}

\section{Analysis of individual learning curves}

As the first 209 biopsies were performed by Operator 1, we split these cases into four groups of 52 patients (the last group included 53 patients). We replicated the same split for the biopsies performed by Operator 2 .

The characteristics of the patients who underwent MRITRUS fusion prostate biopsy by Operator 1 are summarized in Table 1. The median age of the patients was 63 (IQR: 5868) years, the median PSA was $6.8(4.77-9.608) \mathrm{ng} / \mathrm{mL}$ and the median prostate volume was 49 (IQR: 37-67.1) g. Less than a third of the patients had a history of prior prostate biopsy (26.8\%). Positive trends were observed for all outcomes of MRI-TRUS fusion prostate biopsy. There was an increase of $12.52 \%$ in overall $\mathrm{PCa}$ detection rate $(36.53 \%$ vs. $49.05 \%, \mathrm{P}=0.341)$ and of $18.51 \%$ in csPCa detection rate $(21.11 \%$ vs. $39.62 \%, \mathrm{P}=0.134)$ between the first and the last patients. Also, an increasing trend was observed for $\mathrm{PCa}$ and csPCa diagnostic yield in patients with PIRADS $4 / 5$ lesions (36\% vs. $54.16 \%$ for overall $\mathrm{PCa}, \mathrm{P}=0.433$ and $28 \%$ vs. $45.83 \%$ for csPCa, $\mathrm{P}=0.561)$. Furthermore, we observed a difference of $66.67 \%$ in the median percentage of positive biopsy cores/targeted ( $0 \%$ vs. $66.67 \%, \mathrm{P}=0.06)$ and $31.43 \%$ in the median percentage of $\mathrm{PCa}$ tissue on positive cores ( $20 \%$ vs. $51 \%, \mathrm{P}=0.268)$ between the first and the last groups of patients.

The characteristics of the patients who underwent MRI-TRUS fusion prostate biopsy by Operator 2 are summarized in Table 2. The median age of the patients was 65 years (IQR: 61-69), the median PSA was 7.045 (IQR: $5.4-12) \mathrm{ng} / \mathrm{mL}$ and the median prostate volume was 47.59 (IQR: 38.88-77) g. Twenty-three percent of the patients had a history of at least one previous prostate biopsy. During the study period, we observed an increase of $25.44 \%$ in overall $\mathrm{PCa}$ detection rate $(28.84 \%$ vs. $54.28 \%, \mathrm{P}=0.04)$, and $15 \%$ in csPCa detection rate $(25 \%$ vs. $40 \%, \mathrm{P}=0.145)$. A significant increase was seen for overall $\mathrm{PCa}$ detection rate in patients with PIRADS $4 / 5$ lesions $(38.09 \%$ vs. $80.95 \%, \mathrm{P}=0.02)$. The diagnosis rate of csPCa showed a similar increase in this subgroup of patients, from the first until the last $(33.33 \%$ vs. $66.66 \%, \mathrm{P}=0.09)$. The median percentage of positive biopsy cores/targeted and the median percentage of cancer on positive cores were not significantly different between the first and the last groups of patients. 
Table 1 Characteristics and outcomes of the patients who underwent MRI-TRUS fusion biopsy by Operator 1

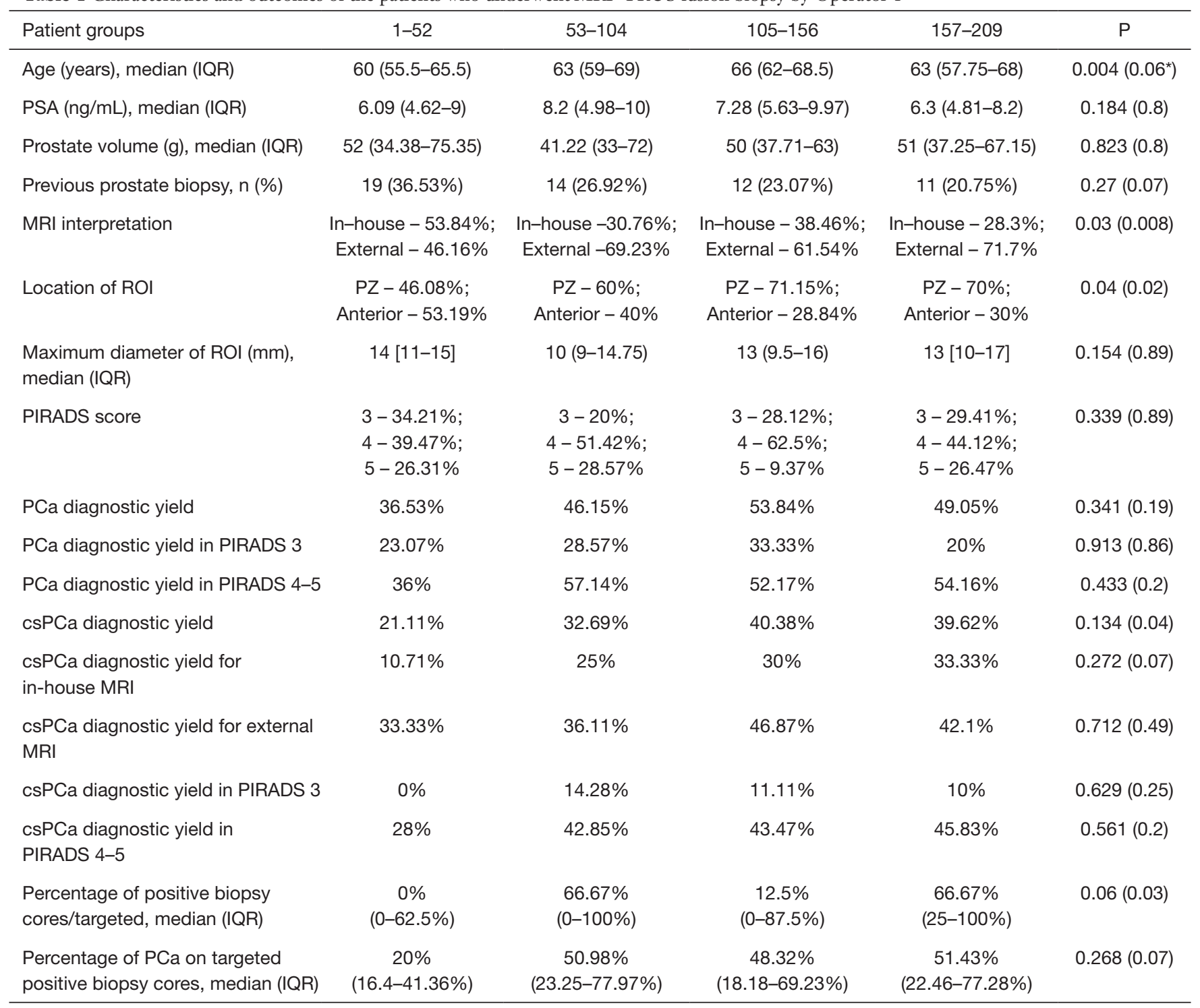

* the second $\mathrm{P}$ value has been obtained by comparing the patients in the first and last group. csPCa, clinically significant prostate cancer; IQR, interquartile range; MRI, magnetic resonance imaging; PCa, prostate cancer; PIRADS, Prostate Imaging-Reporting Data System; PSA, prostate specific antigen; ROI, region of interest; TRUS, transrectal ultrasound.

\section{Comparison of learning curves}

We compared the first 52 procedures performed independently by each operator in order to assess whether there are differences in their learning curves, as they had different backgrounds. We observed no significant differences when comparing the outcome of the biopsy between the two groups (Tables 1-3). Similar results were observed when comparing the last biopsies performed by both operators, with no significant differences in terms of $\mathrm{PCa}$ and csPCa detection rates. Figure 1 illustrates the learning curves of the two operators, showing comparable outcomes.

\section{Impact of experience on $\mathrm{PCa}$ detection rate}

Multivariate analysis adjusted for age, PSA, prostate volume, ROI diameter and PIRADS score showed that for every 52 biopsies performed, the odds of detecting $\mathrm{PCa}$ increase with $51 \%, \mathrm{P}=0.022$ (Table 4). Hosmer Lemeshow goodness 
Table 2 Characteristics and outcomes of the patients who underwent MRI-TRUS fusion biopsy by Operator 2

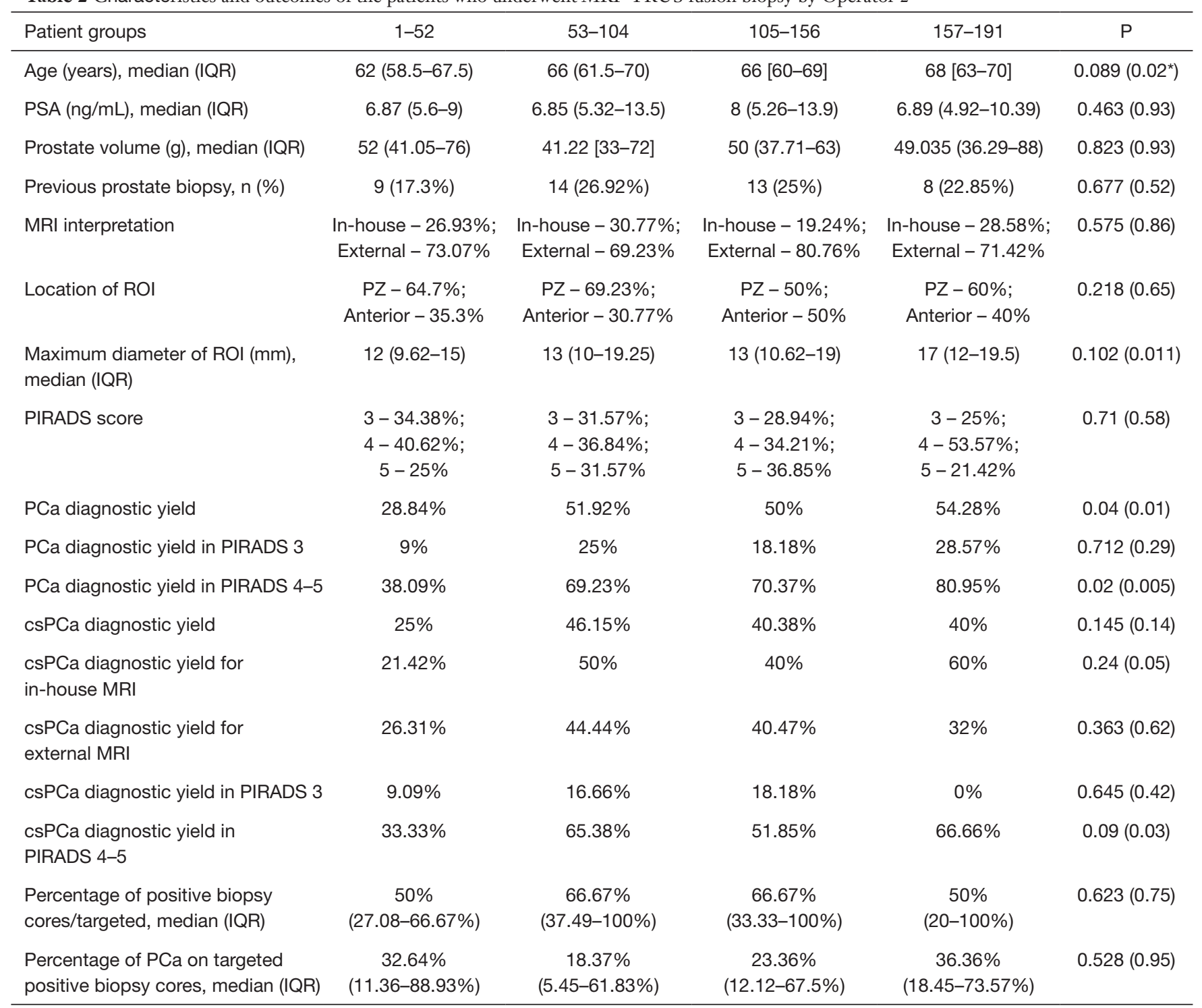

* the second $\mathrm{P}$ value has been obtained by comparing the patients in the first and last group. csPCa, clinically significant prostate cancer; IQR, interquartile range; MRI, magnetic resonance imaging; PCa, prostate cancer; PIRADS, Prostate Imaging-Reporting Data System; PSA, prostate specific antigen; ROI, region of interest; TRUS, transrectal ultrasound.

of fit test has shown a $\mathrm{P}$ value of 0.468 , demonstrating no significant difference between the model's prediction and the actual data. The accuracy of the classification was $76.68 \%$ and area under the receiver operating characteristic curve was 85.85 (95\% CI: 80.7-91.01).

\section{Discussion}

The aim of our study was to assess the change in PCa detection rate over time of two different urologists with distinct background, one being a senior urologist (Operator 1), the second one being a resident (Operator 2).

Analyzing the individual learning curve of Operator 1, we observed an increasing trend in overall PCa diagnostic yield from the first cases until the last (Table 1). This could be due to the fact that overall detection of PCa depends on mastering systematic biopsy. Operator 1 was already experienced in systematic biopsy, thus the learning curve was overcome for this procedure. On the other hand, although the learning curve of Operator 2 replicated the 
Table 3 Comparison of characteristics and biopsy outcomes of the patients who underwent MRI-TRUS fusion prostate biopsy by both Operators in the beginning and the end of the learning curve assessment

\begin{tabular}{|c|c|c|}
\hline Patient details and biopsy outcomes & $\begin{array}{l}\text { First group of patients who } \\
\text { underwent MRI-TRUS fusion biopsy } \\
\text { by Operator } 1 \text { vs. Operator } 2^{\dagger}\end{array}$ & $\begin{array}{l}\text { Last group of patients who } \\
\text { underwent MRI-TRUS fusion biopsy } \\
\text { by Operator } 1 \text { vs. Operator } 2^{+}\end{array}$ \\
\hline Age & 0.04 & 0.01 \\
\hline PSA & 0.183 & 0.312 \\
\hline Previous prostate biopsy & 0.02 & 0.81 \\
\hline MRI interpretation (external vs. in-house) & 0.005 & 0.97 \\
\hline Location of ROI & 0.07 & 0.34 \\
\hline Maximum diameter of ROI & 0.322 & 0.03 \\
\hline PCa diagnostic yield in PIRADS 3 & 0.36 & 0.69 \\
\hline PCa diagnostic yield in PIRADS 4-5 & 0.88 & 0.06 \\
\hline csPCa diagnostic yield & 0.64 & 0.97 \\
\hline csPCa diagnostic yield for in-house MRI & 0.355 & 0.19 \\
\hline csPCa diagnostic yield for external MRI & 0.55 & 0.422 \\
\hline csPCa diagnostic yield in PIRADS 3 & 0.277 & 0.4 \\
\hline csPCa diagnostic yield in PIRADS 4-5 & 0.69 & 0.165 \\
\hline
\end{tabular}

${ }^{\dagger}$, Please see Tables 1 and 2 for all corresponding numeric data. csPCa, clinically significant prostate cancer; MRI, magnetic resonance imaging; PCa, prostate cancer; PIRADS, Prostate Imaging-Reporting Data System; PSA, prostate specific antigen; ROI, region of interest; TRUS, transrectal ultrasound.

one of Operator 1, we identified significant differences in terms of overall PCa detection rate in all and in PIRADS $4 / 5$ patients between the first patients and the last. These differences could be due to the fact that Operator 2 learned in parallel the technique of systematic and MRI-TRUS fusion biopsy. A similar improvement of overall CDR was previously reported, although the final rates are higher [Meng et al. (11): $50 \%$ to $76 \%, \mathrm{P}=0.032$; Gaziev et al. (14): $42 \%$ to $64 \%$ to $81 \%, \mathrm{P}<0.003]$. A positive trend was observed also for csPCa diagnosis rate of both operators in our study $(21.11 \%$ vs. $39.62 \%, \mathrm{P}=0.134$ for Operator $1 ; 25 \%$ vs. $40 \%, \mathrm{P}=0.145$ for Operator 2). Similar rates of csPCa detection rate were reported by Calio et al. $(24.8 \%$ to $36.4 \%, \mathrm{P}<0.0001$ ) (12). One reason for the lower PCa detection rate in our study is that the mpMRI interpretation was heterogenous, coming from various centers, in contrast with Meng et al. (11), who report that a single radiologist assessed all the mpMRIs. Similarly, Calio et al. (12) included only patients who underwent the same protocol for mpMRI in a tertiary center. It was previously shown that mpMRI review by prostate imaging experts downgraded PI-RADS score in $50 \%$ of cases. Thus, one of the causes of targeted biopsy failure could be represented by an inaccurate PIRADS score (15).

Furthermore, there was a positive trend in the percentage of positive biopsy cores/targeted for Operator 1 ( $0 \%$ vs. $66.67 \%, \mathrm{P}=0.06$ ), suggesting an improved targeting. Improvement in the quality of the biopsy cores over time 

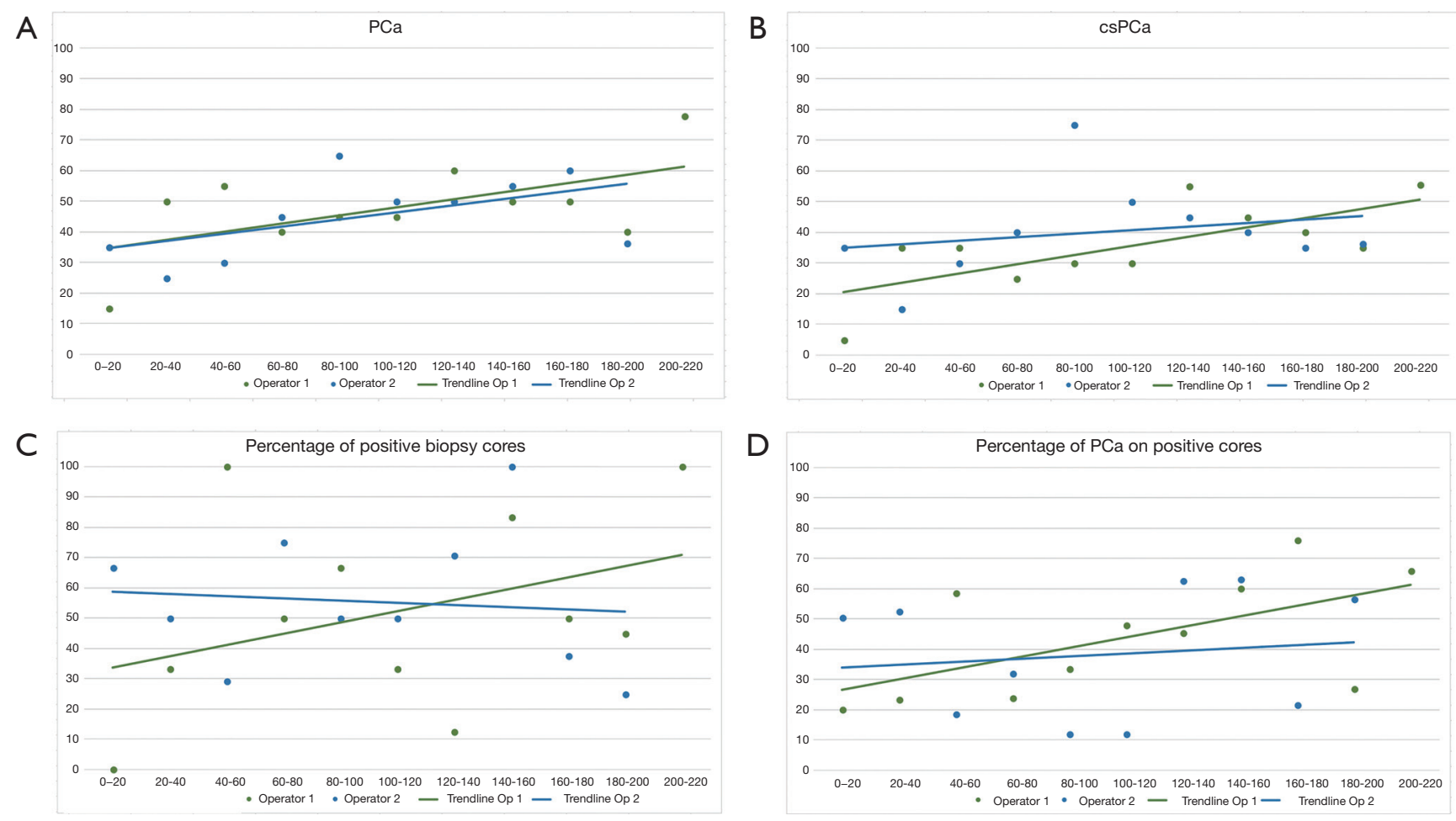

Figure 1 Comparison between the learning curves of MRI-TRUS fusion prostate biopsy of the two operators. (A) Dynamic of overall PCa detection rate during the learning curve of the two operators; (B) dynamic of clinically significant PCa detection rate during the learning curve of the two operators; (C) dynamic of percentage of positive biopsy cores/targeted during the learning curve of the two operators; (D) dynamic of percentage of $\mathrm{PCa}$ on positive cores during the learning curve of the two operators. csPCa, clinically significant prostate cancer; $\mathrm{PCa}$, prostate cancer.

Table 4 Multivariate analysis of factors associated with PCa diagnosis

\begin{tabular}{lccc}
\hline Characteristic & OR adjusted & $95 \% \mathrm{Cl}$ & $\mathrm{P}$ \\
\hline Age (years) & 1.11 & $1.04-1.18$ & 0.002 \\
PSA (ng/mL) & 1.13 & $1.03-1.24$ & 0.011 \\
Prostate volume $(\mathrm{g})$ & 0.95 & $0.93-0.97$ & $<0.001$ \\
ROI diameter $(\mathrm{mm})$ & 1.02 & $0.94-1.1$ & 0.643 \\
PIRADS score & 1.68 & $0.89-3.2$ & 0.113 \\
Experience $^{\dagger}$ & 1.51 & $1.07-2.16$ & 0.022 \\
\hline
\end{tabular}

${ }^{\dagger}$, Experience was quantified as a numerical variable, with a 1-unit increase with every 52 biopsies performed. PCa, prostate cancer; PIRADS, Prostate Imaging-Reporting Data System; PSA, prostate specific antigen; $\mathrm{ROI}$, region of interest.

was also previously reported by Kasabwala et al. (16) in terms of: higher length of prostate tissue, shorter distance between the needle trajectory and target, lower quantity of non-prostatic tissue, lower number of cores that missed prostate entirely (16).

The statistically significant increase of PCa detection after 52 cases shows that the volume of the procedures still represents an important determinant factor for the learning curve, similar to what was previously reported by Meng et al. (11). Regarding csPCa, Stabile et al. (17) observed a similar increase in the diagnosis rate $(53 \%$ to $83 \%)$ after 60 targeted biopsies. Reporting the results for a single urologist with no previous experience in fusion biopsy, Kasabwala et al. (16) showed that improvements in the targeting accuracy are seen up to 98 cases. Further on, the detection rate showed a small insignificant increase and did not reach a plateau during the study (16). Halstuch et al. (18) analyzed both the surgical process (biopsy duration) and patient outcome (PCa CDR) in a cohort of 779 patients who were biopsied by a single urologist. They observed that a plateau was reached after 109 biopsies for procedure duration (45 to $15 \mathrm{~min}, \mathrm{P}<0.001$ ) and 110 cases for CDR 
(35\% to 50\% PCa detection rate for PIRADS 3 lesions) (18). In our study, for both operators, we observed an increase in $\mathrm{PCa}$ detection rate for the first 52 cases, followed by a plateau, suggesting that there might be a learning curve of approximately 52 procedures to reach a stable PCa diagnosis yield (Tables 1,2). In terms of csPCa, there was also an increase in the diagnosis yield up the $104^{\text {th }}$ case for Operator 1, but not for Operator 2. The csPCa diagnosis yield of Operator 2 seems to be stable after the first 52 cases, suggesting a shorter learning curve as compared to Operator 1. Hence, depending on the background of the operator, approximately 50-100 cases seem to be needed to reach a plateau for csPCa detection rate. Our data support the results already published by Halstuch et al. and Kasabwala et al., showing that 98-110 biopsies are needed in order to obtain good quality biopsy results and a reliable detection rate.

An interesting point underlined by Meng et al. (11) is that if the learning curve of targeted biopsy is volume dependent, all operators will achieve similar outcomes with experience. This concept was well illustrated in our study. When comparing the learning curves of the two urologists, we did not observe any significant difference between the first and last cases performed by both of them. A similar report by Mager et al. (19) compared the learning curve of an expert urologist to a novice urologist. Their findings also show no statistical difference between the expert and the novice at the end of their learning curve regarding the CDR. However, a statistical difference was observed for the first cases of the novice, underlining the importance of mentoring by an expert when a novice starts his activity of targeted biopsy. In our study, the Operator 2 benefitted from the expertise of Operator 1 at the beginning of his learning curve, reducing the time needed to standardize the MRI-US fusion technique. Setia et al. (20) showed that a workshop prior to performing fusion biopsy is useful for a urology resident as it improves the knowledge and familiarity with the procedure. Also, participating to an ibook MRI training course has been showed to lead to a significant improvement both in test performance ( $37 \%$ pre-course vs. $57 \%$ post-course, $\mathrm{P}=0.0039$ ) and in confidence in using this imaging technique $(\mathrm{P}=0.01)$ (8). Another important variable is the physician ability to use the information provided by the mpMRI in performing the systematic biopsy. Iwamoto et al. (21) reported that for two urologists with similar backgrounds and experience the CDR of systematic biopsy was significantly higher for patients who had visible lesions on mpMRI ( $43 \% v s$. $73 \%, \mathrm{P}=0.001)$. Therefore, training in mpMRI reading and performing a cognitive-guided systematic biopsy might explain Operator 2 initial higher CDR.

On the other hand, as underlined by Sathianathen et al. (22), there are several external factors such as technological differences, MRI interpretation and quality, experience of the radiologist, pathology assessment and biopsy indication that impact the learning curve and biopsy outcomes, apart from individual experience. Although we found no significant difference between the PCa diagnosis rate in patients with in-house vs external MRI, this is not enough to prove similarity in terms of scan quality and reporting. The lack of assessing the impact of these external factors represents a limitation of our study.

The implementation of an MRI-US fusion biopsy program should be a multidisciplinary team (radiologist, urologist, pathologist) effort in order to obtain the best results. As shown by Urkmez et al. (23), significant improvement in the detection rate of MRI-TRUS fusion biopsy can be observed once expertise is achieved by all team members. As compared to our study, a higher increase in overall and csPCa diagnosis rate was observed by the authors after mastering the procedure, with an almost double CDR from the first 50 patients until the last. Being a transdisciplinary procedure, collaboration is the key to improve outcomes. However, the current paper illustrates the learning curve of MRI-TRUS fusion prostate biopsy from the point of view of the urologist. Performing a good quality procedure entails an exact and accurate targeting. It requires mastering the technique, being able to correctly identify a lesion on the MRI, perform its segmentation, overlapping the MRI and US volumes and overcoming movement errors. Prior experience in prostate mpMRI, such as courses for urologists, and the presence of a mentor could lead to a better start and a shorter learning curve for the urologist.

\section{Conclusions}

Mentoring and prostate MRI reading training allow a novice urologist to demonstrate a good initial PCa detection rate when starting with MRI-US fusion prostate biopsy. About 52 cases were needed to reach a stable PCa and csPCa detection rate in our cohort. An experienced urologist starting on his own could reach the same rates after about 52-104 cases performed. 


\section{Acknowledgments}

Funding: This work was supported by a grant of the Romanian Ministry of Education and Research, CNCS - UEFISCDI, project number PN-III-P1-1.1PD-2019-1237, within PNCDI III.

\section{Footnote}

Reporting Checklist: The authors have completed the STROBE reporting checklist. Available at http://dx.doi. org/10.21037/tau-21-8

Data Sharing Statement: Available at http://dx.doi. org/10.21037/tau-21-8

Peer Review File: Available at http://dx.doi.org/10.21037/ tau-21-8

Conflicts of Interest: All authors have completed the ICMJE uniform disclosure form (available at http://dx.doi. org/10.21037/tau-21-8). The authors have no conflicts of interest to declare.

Ethical Statement: The authors are accountable for all aspects of the work in ensuring that questions related to the accuracy or integrity of any part of the work are appropriately investigated and resolved. The study was conducted in accordance with the Declaration of Helsinki (as revised in 2013) and was approved by the local Hospital Ethical Committee (approval no. 8/20.02.2017). All patients enrolled completed and signed the informed consent form.

Open Access Statement: This is an Open Access article distributed in accordance with the Creative Commons Attribution-NonCommercial-NoDerivs 4.0 International License (CC BY-NC-ND 4.0), which permits the noncommercial replication and distribution of the article with the strict proviso that no changes or edits are made and the original work is properly cited (including links to both the formal publication through the relevant DOI and the license). See: https://creativecommons.org/licenses/by-nc-nd/4.0/.

\section{References}

1. 1. Mottet N, Cornford P, van den Bergh RCN, et al. EAU-EANM-ESTRO-ESUR-SIOG Guidelines on Prostate Cancer. EAU Guidelines Office, Arnhem, 2020.
2. Tooker GM, Truong H, Pinto PA, et al. National survey of patterns employing targeted MRI/US guided prostate biopsy in the diagnosis and staging of prostate cancer. Curr Urol 2019;12:97-103.

3. Wegelin O, van Melick HHE, Hooft L, et al. Comparing three different techniques for magnetic resonance imaging-targeted prostate biopsies: a systematic review of in-bore versus magnetic resonance imaging-transrectal ultrasound fusion versus cognitive registration. Is there a preferred technique? Eur Urol 2017;71:517-31.

4. Arsov C, Rabenalt R, Quentin M, et al. Comparison of patient comfort between MR-guided in-bore and MRI/ultrasound fusion-guided prostate biopsies within a prospective randomized trial. World J Urol 2016;34:215-20.

5. Eineluoto JT, Järvinen P, Kilpeläinen T, et al. Patient experience of systematic versus fusion prostate biopsies. Eur Urol Oncol 2018;1:202-7.

6. Venderink W, Govers TM, De Rooij M, et al. Costeffectiveness comparison of imaging-guided prostate biopsy techniques: Systematic transrectal ultrasound, direct in-bore MRI, and image fusion. AJR Am J Roentgenol 2017;208:1058-63.

7. Cerantola Y, Dragomir A, Tanguay S, et al. Costeffectiveness of multiparametric magnetic resonance imaging and targeted biopsy in diagnosing prostate cancer. Urol Oncol 2016;34:119.e1-9.

8. Wang NN, Fan RE, Ghanouni P, et al. Teaching urologists "How to read multiparametric prostate MRIs using PIRADSv2": results of an iBook pilot study. Urology 2019;131:40-5.

9. Westhoff N, Siegel F, Peter C, et al. Defining the target prior to prostate fusion biopsy: the effect of MRI reporting on cancer detection. World J Urol 2019;37:327-35.

10. Venderink W, de Rooij M, Sedelaar JPM, et al. Elastic versus rigid image registration in magnetic resonance imaging-transrectal ultrasound fusion prostate biopsy: a systematic review and meta-analysis. Eur Urol Focus 2018;4:219-27.

11. Meng X, Rosenkrantz AB, Huang R, et al. The institutional learning curve of magnetic resonance imaging-ultrasound fusion targeted prostate biopsy: temporal improvements in cancer detection in 4 years. $\mathrm{J}$ Urol 2018;200:1022-9.

12. Calio B, Sidana A, Sugano D, et al. Changes in prostate cancer detection rate of MRI-TRUS fusion vs systematic biopsy over time: Evidence of a learning curve. Prostate 
Cancer Prostatic Dis 2017;20:436-41.

13. Andras I, Crisan D, Cata E, et al. MRI-TRUS fusion guided prostate biopsy - initial experience and assessment of the role of contralateral lobe systematic biopsy. Med Ultrason 2019;21:37-44.

14. Gaziev G, Wadhwa K, Barrett T, et al. Defining the learning curve for multiparametric magnetic resonance imaging (MRI) of the prostate using MRI-transrectal ultrasonography (TRUS) fusion-guided transperineal prostate biopsies as a validation tool. BJU Int 2016;117:80-6.

15. Cash H, Günzel K, Maxeiner A, et al. Prostate cancer detection on transrectal ultrasonography-guided random biopsy despite negative real-time magnetic resonance imaging/ultrasonography fusion-guided targeted biopsy: reasons for targeted biopsy failure. BJU Int 2016;118:35-43.

16. Kasabwala K, Patel N, Cricco-Lizza E, et al. The learning curve for magnetic resonance imaging/ultrasound fusionguided prostate biopsy. Eur Urol Oncol 2019;2:135-40.

17. Stabile A, Dell'Oglio P, Gandaglia G, et al. Not all multiparametric magnetic resonance imaging-targeted biopsies are equal: the impact of the type of approach and operator expertise on the detection of clinically significant

Cite this article as: Cata ED, Van Praet C, Andras I, Kadula P, Ognean R, Buzoianu M, Leucuta D, Caraiani C, Tamas-Szora A, Decaestecker K, Coman I, Crisan N. Analyzing the learning curves of a novice and an experienced urologist for transrectal magnetic resonance imaging-ultrasound fusion prostate biopsy. Transl Androl Urol 2021;10(5):1956-1965. doi: 10.21037/tau-21-8 prostate cancer. Eur Urol Oncol 2018;1:120-8.

18. Halstuch D, Baniel J, Lifshitz D, et al. Characterizing the learning curve of MRI-US fusion prostate biopsies. Prostate Cancer Prostatic Dis 2019;22:546-51.

19. Mager R, Brandt MP, Borgmann H, et al. From novice to expert: analyzing the learning curve for MRI-transrectal ultrasonography fusion-guided transrectal prostate biopsy. Int Urol Nephrol 2017;49:1537-44.

20. Setia S, Feng C, Coogan C, et al. Urology residents' experience with simulation: initial evaluation of MRI/US fusion biopsy workshop. Urology 2019;134:51-5.

21. Iwamoto H, Yumioka T, Yamaguchi N, et al. The efficacy of target biopsy of suspected cancer lesions detected by magnetic resonance imaging and/or transrectal ultrasonography during initial prostate biopsies: Comparison of outcomes between two physicians. Yonago Acta Med 2014;57:53-8.

22. Sathianathen NJ, Warlick CA, Soubra A, et al. Difference in MRI-guided biopsy cancer detection rates between individual clinicians. Urol Oncol 2019;37:299.e1-299.e6.

23. Urkmez A, Ward JF, Choi H, et al. Temporal learning curve of a multidisciplinary team for magnetic resonance imaging/transrectal ultrasonography fusion prostate biopsy. BJU Int 2021;127:524-7. 